\title{
BIBECHANA
}

A Multidisciplinary Journal of Science, Technology and Mathematics ISSN 2091-0762 (Print), 2382-5340 (Online)

Journal homepage: http://nepjol.info/index.php/BIBECHANA

Publisher: Research Council of Science and Technology, Biratnagar, Nepal

\section{On zero-free regions for the derivative of a polynomial}

\section{B .A. Zargar* and A . W. Manzoor}

Department of Mathematics, University of Kashmir Hazratbal Srinagar 190006, India

*Email: bazargar@gmail.com

Article history: Received 27 June, 2016; Accepted 17 August, 2016

DOI: http://dx.doi.org/10.3126/bibechana.v14i0.15521

This work is licensed under the Creative Commons CC BY-NC License.

https://creativecommons.org/licenses/by-nc/4.0/

(c) (1) (9)

\section{Abstract}

Let $\mathrm{P}_{\mathrm{n}}$ denote the set of all polynomials of the form $p(z)=z \prod_{j=1}^{n-1}\left(z-z_{j}\right)$ with $\left|z_{j}\right| \geq 1, \quad 1 \leq j \leq n-1$. In this paper we shall obtain some zero-free regions for the derivative of a polynomial.

Keywords: Zero-free regions; Critical points; Sendov's Conjecture.

\section{Introduction}

Let us suppose that $\mathrm{p}(\mathrm{z})$ is an $\mathrm{n}^{\text {th }}$ degree polynomial which has all its zeros in the unit disk $|z| \leq 1$, then all the critical points of $\mathrm{p}(\mathrm{z})$ also lie in the same disk $|z| \leq 1$. This is in fact the wellknown Theorem which was implied in a note of Gauss dated 1836 and proved explicitly by Lucas dated 1874 (see also Marden [1].

Now instead of considering the relative position of all the zeros and critical points of $\mathrm{p}(\mathrm{z})$, let us choose any one zero $z_{0}$ of $p(z)$ and ask: At most how far from $z_{0}$ does the nearest critical point lie ? A possible answer to this question is given by the following :Conjecture: “If $\mathrm{p}(\mathrm{z})$ is an $\mathrm{n}^{\text {th }}$ degree polynomial having all its zeros in the unit disk $|z| \leq 1$ and if $z_{0}$ is any one such zero, then at least one critical point of $\mathrm{p}(\mathrm{z})$ lie in the disk $\left|z-z_{0}\right| \leq 1$." This conjecture was included in the collection of Research Problems in Function Theory published in 1967 by professor Hayman [2], (see also [3]). Since it had been brought to Hayman's attention by professor Ilyeff. It became 
known as "Ilyeff's Conjecture". Actually conjecture was due to a Bulgarian mathematician B. Sendov. In connection with this conjecture Brown [4] posed the following problem.

Let $Q_{n}$ denote the set of all complex polynomials of the form $p(z)=z \prod_{j=1}^{n-1}\left(z-z_{j}\right)$ with $\left|z_{j}\right| \geq 1, \quad 1 \leq j \leq n-1$. Find the best constant $C_{n}$ such that $p^{\prime}(z)$ does not vanish in $|z| \leq C_{n}$ for all $p \in Q_{n}$.

Brown observed that if $p(z)=z(z-1)^{n-1}$ then $p^{\prime}\left(\frac{1}{n}\right)=0$ and conjectured that $C_{n}=\frac{1}{n}$.

Recently Aziz and Zargar [5] settled this conjecture.

Theorem1.1. Let $p(z)=z \prod_{k=1}^{n-1}\left(z-z_{k}\right)$ be a polynomial of degree $\mathrm{n}$ with $\left|z_{k}\right| \geq 1, \quad 1 \leq k \leq n-1$, then $p^{\prime}(z)$ does not vanish in the disk $|z|<\frac{1}{n}$.

The result is best possible for the polynomial $p(z)=z\left(z-e^{i \alpha}\right)^{n-1}, 0 \leq \alpha<2 \pi$.

First we shall prove the following interesting result which provides the zero free regions for the second derivative of polynomial

$p(z)=z^{m} \prod_{k=1}^{n-m}\left(z-z_{j}\right)$

Theorem 1.2. If $p(z)=z^{m} \prod_{j=1}^{n-m}\left(z-z_{j}\right)$ where $\left|z_{j}\right| \geq 1, j=1,2, \ldots \ldots \ldots . n-m$, then $\quad$ the polynomial $p^{\prime \prime}(z)$ does not vanish in

$0<|z|<\frac{m(m-1)}{n(n-1)}$.

Taking $\mathrm{m}=2$ we get

Corollary 1.If $\quad p(z)=z^{2} \prod_{j=1}^{n-2}\left(z-z_{j}\right)$ where $\left|z_{j}\right| \geq 1, j=1,2, \ldots \ldots \ldots . . n-2$, then the polynomial $p^{\prime \prime}(z)$ does not vanish in

$0<|z|<\frac{2}{n(n-1)}$.

It is clearly of interest to known that a zero free region for the polynomial $p^{m}(z)$ where

$$
p(z)=z^{m} \prod_{j=1}^{n-m}\left(z-z_{j}\right)
$$

In this direction, we prove the following interesting results: 
Theorem1.3. Let

$$
p(z)=z^{m} \prod_{j=1}^{n-m}\left(z-z_{j}\right)
$$

be a polynomial of degree $\mathrm{n}$, with $\left|z_{j}\right| \geq 1, j=1,2, \ldots \ldots \ldots . n-m$, then the polynomial $p^{m}(z)$ does not vanish in the disk

$$
|z|<\frac{m !}{n(n-1) \ldots .(n-m+1)}
$$

Remark 1. If $\mathrm{m}=1$, then we get Theorem 1.1.

For the proofs of these theorems we need the following result which is due to Aziz and Zagar [5].

Lemma: Let $p(z)=z^{m} \prod_{j=1}^{n-m}\left(z-z_{j}\right)$ where $\left|z_{j}\right| \geq 1, \quad 1 \leq j \leq n-m$, then $p^{\prime}(z)$ does

not vanish in $0<|z|<\frac{m}{n}$.

\section{Proofs of Theorems}

Proof of Theorem 1.2. We write,

$$
p(z)=z^{m} Q(z)
$$

where

$$
Q(z)=\prod_{j=1}^{n-m}\left(z-z_{j}\right),\left|z_{j}\right| \geq 1, j=1,2, \ldots \ldots n-m .
$$

By above lemma, the polynomial

$$
\begin{aligned}
& p^{\prime}(z)=z^{m} Q^{\prime}(z)+m z^{m-1} Q(z) \\
& =z^{m-1} R(z),
\end{aligned}
$$

where

$$
R(z)=z Q^{\prime}(z)+m Q(z)
$$

does not vanish in $0<|z|<\frac{m}{n}$.

Replacing zby $\frac{m}{n} z$, it follows that the polynomial,

$$
\begin{aligned}
S(z) & =p^{\prime}\left(\frac{m}{n} z\right) \\
& =\left(\frac{m}{n}\right)^{m-1} z^{m-1} R\left(\frac{m}{n} z\right)
\end{aligned}
$$

does not vanish in $0<|z|<1$, so that all the zeros of $R\left(\frac{m}{n} z\right)$ lie in $|z| \geq 1$. 
Using the above lemma again and noting that $S(z)$ is a polynomial of degree $n-1$, it follows that $S^{\prime}(z)$ does not vanish in

$$
0<|z|<\frac{m-1}{n-1}
$$

Or equivalently,

$$
p^{\prime \prime}\left(\frac{m}{n} z\right)
$$

does not vanish in

$$
0<|z|<\frac{m-1}{n-1}
$$

Replacing $\mathrm{z}$ by $\frac{m}{n} z$, it follows that

$$
\begin{aligned}
p^{\prime \prime}(z) & =z^{m-1} R^{\prime}(z)+(m-1) z^{m-2} R(z) \\
& =z^{m-2}\left(z R^{\prime}(z)+(m-1) R(z)\right) \\
& =z^{m-2} T(z)
\end{aligned}
$$

where

$$
T(z)=z R^{\prime}(z)+(m-1) z R(z)
$$

does not vanish in

$$
0<|z|<\frac{m-1}{n-1}
$$

This completes the proof of Theorem 1.2.

Proof of Theorem 1.3. By hypothesis,

where

$$
p(z)=z^{m} Q(z)
$$

$$
Q(z)=\prod_{j=1}^{n-m}\left(z-z_{j}\right),\left|z_{j}\right| \geq 1, j=1,2, \ldots \ldots . n-m .
$$

By the above lemma, the polynomial $p^{\prime}(z)$ does not vanish in

$$
0<|z|<\frac{m}{n} \text {. }
$$

Therefore Theorem 1.2 yeilds that

$$
p^{\prime \prime}(z)=z^{n-2} T(z)
$$

where

$$
T(z)=z R^{\prime}(z)+(m-1) z R(z)
$$

does not vanish in

$$
0<|z|<\frac{m(m-1)}{n(n-1)} .
$$

Replacing $\mathrm{z}$ by $\frac{m(m-1)}{n(n-1)} z$ it follows that 


$$
\begin{aligned}
& U(z)=p^{\prime \prime}\left(\frac{m(m-1)}{n(n-1)} z\right) \\
& =\left(\frac{m(m-1)}{n(n-1)} z\right)^{n-2} T\left(\frac{m(m-1)}{n(n-1)} z\right)
\end{aligned}
$$

does not vanish in $0<|z|<1$, so that all the zeros of $T\left(\frac{m(m-1)}{n(n-1)} z\right)$ lie in $|z| \geq 1$.Applying the above lemma again and noting that $\mathrm{U}(\mathrm{z})$ is a polynomial of degree $\mathrm{n}-2$, thus it implies that $U^{\prime}(z)$ does not vanish in $0<|z|<\frac{m-2}{n-2}$.

Or equivalently,

$$
p^{\prime \prime \prime}\left(\frac{m(m-1)}{n(n-1)} z\right)
$$

does not vanish in

$$
0<|z|<\frac{m-2}{n-2}
$$

Replacing $\mathrm{z}$ by $\frac{n(n-1)}{m(m-1)} z$ it follows that,

$$
\begin{aligned}
p^{\prime \prime \prime}(z) & =z^{n-2} T^{\prime}(z)+(n-2) z^{n-3} T(z) \\
& =z^{n-3}\left(z T^{\prime}(z)+(n-2) T(z)\right) \\
& =z^{n-3} V(z),
\end{aligned}
$$

where

$$
V(z)=z T^{\prime}(z)+(n-2) T(z)
$$

does not vanish in $0<|z|<\frac{m(m-1)(m-2)}{n(n-1)(n-2)}$.

In a similar way we see that the polynomial

$$
\begin{aligned}
p^{i v}(z)= & z^{n-3} V^{\prime}(z)+(n-3)+(n-3) z^{n-4} V(z) \\
& =z^{n-4} W(z)
\end{aligned}
$$

where,

$$
W(z)=z V^{\prime}(z)+(n-3) V(z)
$$

does not vanish in

$$
0<|z|<\frac{m(m-1)(m-2)(m-3)}{n(n-1)(n-2)(n-3)} .
$$

Proceeding in this way and noting that $m$ and $n$ are positive integers it follows that the polynomial does not vanish in

$$
|z|<\frac{m(m-1) \ldots \ldots .2 .1}{n(n-1) \ldots .(n-m+1)}=\frac{m !}{n(n-1) \ldots . .(n-m+1)}
$$

Which proves Theorem 1.3. 
B .A. Zargar and A. W. Manzoo / BIBECHANA 14 (2017) 48-53 : RCOST p.53 (Online Publication: Dec., 2016)

\section{References}

[1] M. Marden, Geometry of polynomials, Math. Survey's No.3, American Math.Soc. (1966).

[2] W. K. Hayman, Research Problems in Function Theory, London (1967) pp. 1-25.

[3] Q. I. Rahman and G. Schmesser's Analytic Theory of polynomials, Oxford University Press,New York (2002).

[4] J. E. Brown, On thellief-Sendov Conjecture, Pacific J.Math.135 (1988) 223-232. http://dx.doi.org/10.2140/pjm.1988.135.223

[5] A. Aziz and B.A. Zargar, On the critical points of a polynomial, Bull. Austral. Math. Soc.57 (1998) 173-174.

http://dx.doi.org/10.1017/S000497270003152X 